\title{
Enfoques del ejercicio terapéutico sobre la espasticidad en miembro inferior post-Enfermedad Cerebro Vascular: revisión sistemática
}

\author{
Approaches of therapeutic exercise over lower limb \\ spasticity after stroke: a systematic review
}

\author{
Odair A Bacca ${ }^{1}$, María S Patiño ${ }^{1}$, Esperanza Herrera ${ }^{1}$, José A Barela ${ }^{2}$
}

Forma de citar: Bacca OA, Patiño MS, Herrera E, Barela JA. Enfoques del ejercicio terapéutico sobre la espasticidad en miembro inferior post-Enfermedad Cerebro Vascular: revisión sistemática.Rev Univ Ind Santander Salud. 2017; 49(2): 364-378. DOI: http://dx.doi.org/10.18273/revsal.v49n2-2017009 (c) (1)

\section{RESUMEN}

Introducción: En la enfermedad cerebro vascular uno de los principales problemas es la espasticidad, que compromete el control postural, la funcionalidad y la calidad de vida. Para lograr la mayor recuperación funcional, se han utilizado los enfoques neuromusculares del ejercicio terapéutico (Ej: Rood, Bobath, Brunnstrom y la Facilitación Neuromuscular Propioceptiva), sin embargo, existe controversia sobre la eficacia de estas intervenciones. Objetivo: Realizar un análisis crítico de la evidencia científica disponible sobre el efecto de los enfoques tradicionales en la espasticidad de los miembros inferiores en personas post-Enfermedad Cerebro Vascular. Métodos: Se incluyeron ensayos clínicos controlados de las bases de datos PUBMED, MEDLINE, PEDro, CINAHL, SCIENCEDIRECT y SCOPUS. La calidad metodológica de los artículos incluidos se evaluó por medio de la escala PeDro. Resultados: Once estudios cumplieron con los criterios de inclusión, obteniendo una calidad metodológica de intermedia a alta. Entre las limitaciones de los estudios revisados se destacan principalmente la ausencia del cálculo del tamaño de muestra, de enmascaramiento de los evaluadores, pérdida de participantes, muestras heterogéneas, carencia de protocolos estandarizados y diversidad de variables evaluadas. Conclusión: El tratamiento fisioterapéutico para disminuir la espasticidad puede ser efectivo aplicado entre tres y cinco días por semana, durante 40 minutos. Sin embargo, estos resultados están soportados en estudios con limitaciones metodológicas y dificultades en su comparación. Por ello, se requieren nuevos estudios para evaluar la eficacia de dichos enfoques, con el fin implementar intervenciones fisioterapéuticas basadas en la mejor evidencia, que contribuyan en una mejor calidad de vida de esta población.

Palabras clave: Terapia por ejercicio, accidente cerebrovascular, espasticidad muscular, fisioterapia, rehabilitación.

1. Universidad Industrial de Santander. Bucaramanga, Colombia.

2. Universidad de Cruzeiro del Sur. Sao Paulo. Brasil.

Correspondencia: Odair Alfonso Bacca Ramírez. Dirección: Carrera 32 29-31 Edificio Orlando Díaz, P. 1 Escuela de Fisioterapia. Correo electrónico: odairbacca@gmail.com. Teléfono: +7 3163563987. 


\section{ABSTRACT}

Introduction: Spasticity due to a stroke is one of the major changes that leads to sensorimotor deficits, causing postural control and functional capability deficits. In order to achieve the best possible functional recovery, several neuromuscular therapeutic exercise approaches have been employed (eg Rood, Bobath, Brunnstrom and proprioceptive neuromuscular facilitation), but still there are many doubts about the effectiveness of these interventions. Purpose: To perform a critical analysis of the available scientific evidence on the effect of the traditional approaches in spasticity recovery of the lower limbs in post-stroke individuals. Methods: Randomized controlled Trials available in PubMed, MEDLINE, PEDro, CINAHL, and Scopus data SCIENCEDIRECT bases were included in this study. The methodological quality of included articles was evaluated by the PEDro scale. Results: Eleven studies were included, obtaining a methodological quality of intermediate to high. The main observed limitations of the analyzed studies were: the absence of sample size estimation, the lack of blind evaluators, loss of participants, heterogeneous samples, lack of standardized protocols and diversity of employed variables. Conclusions: Based upon the results, it can be suggested that spasticity reduction can be effective applying from three to five days interventions per week, lasting 40 minutes. However, such suggestions are supported by studies with methodological limitations and employing different procedures that prevent a direct comparison among the studies. Therefore new studies are required to evaluate the effectiveness of such approaches, to implement physiotherapy interventions based on the scientific evidences that would further contribute to a better quality of life of post-stroke individuals.

Keywords: Exercise therapy, stroke, muscle spasticity, physical therapy modalities, rehabilitation.

\section{INTRODUCCIÓN}

La Enfermedad Cerebro Vascular (ECV) es la pérdida repentina de la función neurológica, causada por la interrupción del flujo sanguíneo al cerebro, usualmente debido al bloqueo de un coágulo (isquemia) o la ruptura de un vaso (hemorragia) ${ }^{1}$. En Estados Unidos, la ECV afecta a 795.000 personas al año ${ }^{2}$, mientras en Colombia los estudios de prevalencia muestran un estimado entre 300 y 550 casos por 100.000 habitantes $^{3}$.

Una de las manifestaciones de la ECV es la espasticidad, la cual según el proyecto SPASM (siglas en inglés de Support Programme for Assembly of a database for Spasticity Measurement $)^{4}$, se define como "la alteración del control sensoriomotor derivada de una lesión de Neurona Motora Superior (NMS), que se manifiesta con una activación muscular intermitente, involuntaria y sostenida", definición que será adoptada en esta revisión.

Esta perspectiva de la espasticidad enfatiza en la interacción de la información sensorial y motora que regula la actividad muscular ${ }^{4}$. Además, considera los efectos de la información sensorial anormal sobre el control motor, la presencia de actividad refleja liberada durante el reposo y el movimiento, especialmente durante actividades funcionales 5 .

El inicio de la espasticidad post-ECV es muy variable y puede manifestarse a corto, mediano o largo plazo, sin evidencia concluyente sobre las causas o factores desencadenantes ${ }^{6}$. En diversos estudios ${ }^{7-9}$ el tiempo de aparición de la espasticidad se ha establecido con mayor frecuencia entre el primer y el tercer mes post-ECV, con una prevalencia entre el 19 y $22 \%$, sin embargo puede incrementarse en la fase crónica aproximadamente hasta los 18 meses, tiempo en el cual, los autores mencionan mayores cambios biomecánicos que neurales en la estructura del músculo ${ }^{10,11}$.

La espasticidad tiene una prevalencia en el miembro inferior post-ECV de 40-600/100.000 e incidencia de 30-485/100.000; la cual genera un impacto negativo en la movilidad, funcionalidad y la calidad de vida ${ }^{12}$. Los estudios han mostrado que seis meses post-ECV entre el $60-80 \%$ de los pacientes caminan independientemente y el $26 \%$ son dependientes en las $\mathrm{AVD}^{13,14}$, por lo cual, el paciente puede requerir de ayudas funcionales o la supervisión de un cuidador ${ }^{15}$. Adicionalmente, se ha encontrado una disminución significativa en pacientes con espasticidad de la funcionalidad (mediana Índice de Barthel 63,3 puntos), comparado con pacientes sin espasticidad (mediana 80,$6 ; p=0.07)^{7}$.

La disminución de la movilidad articular del cuello de pie generada por la espasticidad en personas post-ECV se ha correlacionado inversamente con la limitación en la ejecución de los traslados de sedente a de pie y viceversa $(\mathrm{r}=-0.80)^{16}$. Además, estos problemas repercuten en el control y la coordinación de la extremidad durante la marcha ${ }^{17,18}$ generando dependencia funcional y riesgos de posibles caídas ${ }^{12}$. Schmid, et al. ${ }^{19}$, encontraron que el $36 \%$ de las caídas 
en personas post-ECV sucedió mientras realizaban AVD como aseo y vestido, el $21 \%$ reportó caídas caminando o subiendo y bajando escaleras, mientras el $34 \%$ tropezaron o se resbalaron.

Durante el proceso de rehabilitación, la intervención fisioterapéutica se realiza mediante la utilización de diversos enfoques de tratamiento como el ejercicio terapéutico, el entrenamiento funcional y las modalidades físicas $^{20,21}$, los cuales han sido desarrollados con el fin de facilitar la recuperación funcional de los pacientes.

Algunas intervenciones se centran solamente en un enfoque, en tanto que otros tratamientos son eclécticos y utilizan varios enfoques del ejercicio. Los enfoques neuromusculares [Rood, Bobath, Brunnstrom y la Facilitación Neuromuscular Propioceptiva (FNP) $]^{21,22}$ se basan en la estimulación sensorial, la utilización de diversas posturas que facilitan el movimiento, el posicionamiento para inhibir los patrones anormales y la repetición para mejorar el aprendizaje motor ${ }^{23,24}$. Estos enfoques de tratamiento se basan en mecanismos neurofisiológicos que implican la facilitación, inhibición y activación, los cuales aumentan o disminuyen la actividad neuronal para promover o retrasar una respuesta muscular por estimulación de los mecanorreceptores musculares, cutáneos y mecanismos supraespinales ${ }^{24,25}$.

En pacientes post-ECV, los enfoques neuromusculares utilizan el estiramiento, los patrones inhibitorios de movimiento, así como la estimulación y reeducación de los patrones antagonistas al espástico; con el fin de mejorar la movilidad, la contracción muscular, y el desempeño en actividades funcionales ${ }^{26-28}$. Para evaluar el efecto de estas intervenciones, en los estudios se han utilizado escalas funcionales, sensorio-motoras, de balance, así como el análisis de patrones como la marcha y el paso de sedente a de píe $e^{4,29,30}$. Igualmente para determinar los cambios en la espasticidad se utilizan mediciones del tono muscular y del desempeño muscular tales como el patrón de activación electromiográfica, el reflejo $\mathrm{H}$ o el torque muscular ${ }^{31,32}$.

Los niveles de evidencia del ejercicio terapéutico han generado controversia en cuanto al efecto de las intervenciones y las limitaciones de las revisiones previas disponibles en la literatura. Pollock, et $\mathrm{al}^{21}$. en 2007 realizaron una revisión sistemática, comparando enfoques de intervención con énfasis en patrones inhibitorios y posicionamiento, estiramiento mantenido, Facilitación Neuromuscular Propioceptiva (FNP), reaprendizaje motor y un enfoque mixto, sin encontrar diferencias estadísticamente significativas en las evaluaciones de recuperación funcional y la función motora ( $p=0,82$ y 0,80 , respectivamente), lo cual evidencia la importancia de utilizar un enfoque ecléctico, ya que ningún enfoque por separado se ha mostrado superior a los otros ${ }^{33,34}$.

Por lo anterior, esta revisión tiene por objetivo analizar críticamente la evidencia científica disponible sobre el efecto de los enfoques del ejercicio terapéutico en la espasticidad de Miembro Inferior (MI) en personas postECV, así como los parámetros de tratamiento utilizados y explicar los posibles mecanismos fisiológicos que fundamentan las intervenciones.

\section{MÉTODOS}

\section{Tipos de estudios}

Fueron incluidos Ensayos Clínicos Controlados (ECC) realizados en humanos, con asignación aleatoria de los participantes. Se incluyeron ensayos con o sin enmascaramiento de los participantes, fisioterapeutas y evaluadores.

\section{Tipos de participantes}

Se incluyeron ECC con una población de estudio conformada por adultos (rango de edad entre 40 y 90 años), con diagnóstico de ECV isquémica o hemorrágica en etapa aguda y crónica.

\section{Tipos de intervenciones}

Se incluyeron los enfoques neuromusculares de rehabilitación: Rood, Bobath, Brunnstrom y la Facilitación Neuromuscular Propioceptiva (FNP) sobre la espasticidad del Miembro Inferior (MI). Se excluyeron los enfoques de rehabilitación cuyo objetivo fue promover la recuperación y funcionalidad del miembro superior.

\section{Tipos de medidas de resultados}

Las evaluaciones de resultados definidas fueron: tono muscular, movilidad articular, desempeño muscular, función motora, balance, características de la marcha, funcionalidad y calidad de vida.

\section{Métodos de búsqueda para la identificación de los resultados}

Se incluyeron ensayos publicados en idioma inglés. 


\section{Búsqueda electrónica}

Se realizó la búsqueda de artículos publicados desde el 1 de enero del 2000 al 31 de julio de 2016 en las bases de datos PUBMED, MEDLINE, PEDro, CINAHL, SCIENCEDIRECT y SCOPUS. La búsqueda se hizo por título y resumen utilizando los siguientes términos: stroke AND (rehabilitation OR physical therapy OR stretching effect OR bobath method OR bobath approach OR rood approach OR brunnstrom approach OR propioceptive neuromuscular facilitation).

\section{RESULTADOS}

\section{Descripción de los estudios}

\section{Resultados de la búsqueda bibliográfica}

Utilizando los términos de búsqueda se obtuvieron 3009 resultados en las bases de datos. Se realizó un screening por título y autor, detectando 629 duplicados los cuales fueron excluidos. Adicionalmente se excluyeron 2203 artículos cuyo título no estaba relacionado con la temática de interés de esta revisión y 155 por no estar disponibles en texto completo. De los artículos restantes se hizo la lectura del resumen y se excluyó un artículo porque el tratamiento enfatizaba en tareas funcionales y 10 más debido a que no se tuvieron en cuenta como variables de respuesta el tono muscular, la movilidad articular, el desempeño muscular, la función motora, el balance, las características de la marcha, la funcionalidad o la calidad de vida. Así, la búsqueda bibliográfica concluyó con 11 ECC $^{35-45}$ Figura 1.

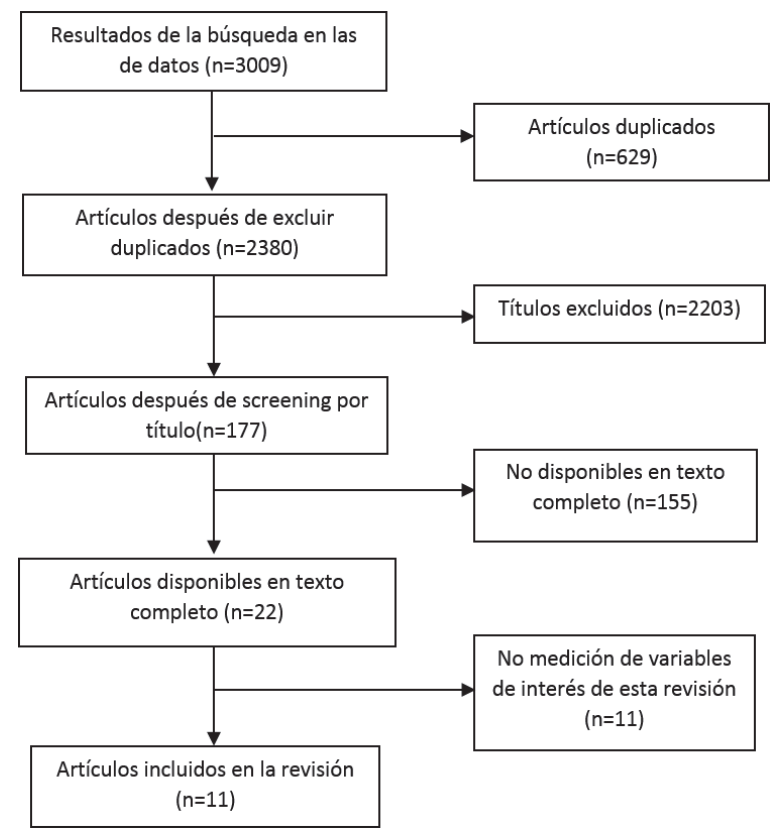

Figura 1. Flujograma de los estudios incluidos en la revisión.
La Tabla 1 muestra los artículos incluidos en la revisión describiendo el objetivo, la población de estudio, el tipo de tratamiento, las variables de salida y los resultados. Así mismo, en la Tabla 2 se describe el análisis metodológico de cada uno de ellos.

La calidad metodológica de los artículos incluidos se evaluó por medio de la escala PeDro, la cual está basada en la lista Delphi desarrollada por Verhagen, et $a{ }^{46}$ en el Departamento de Epidemiología, de la Universidad de Maastricht. La escala incluye 11 ítems y 10 de ellos son tenidos en cuenta para la calificación, para un puntaje total de 10 que significa una excelente metodología del estudio $^{47}$. En la evaluación de la calidad metodológica, los estudios con puntajes entre 7 y 10 fueron considerados de alta calidad metodológica, entre 5 y 6 de calidad intermedia y entre 0 y 4 de baja calidad Tabla 2.

Los estudios incluidos obtuvieron puntajes en un rango entre 6-9, nueve de alta calidad y dos con una calidad metodológica intermedia; sin embargo, en diez de los estudios no se tuvo en cuenta el enmascaramiento del participante, en ocho del terapeuta tratante y en dos de los evaluadores. El puntaje máximo fue de 9 en solamente uno de los estudios $^{36}$ y el puntaje mínimo fue de 6 para los estudios de Fang, et al. ${ }^{38}$ del 2003 y Pohl, et al. ${ }^{43}$ del 2007 Tabla 2.

Dentro de las limitaciones metodológicas encontradas en los estudios revisados se destaca principalmente la ausencia del cálculo del tamaño de muestra, además, en dos estudios ${ }^{38,41}$ al menos una de las variables de salida analizadas fue estadísticamente diferente en la línea de base y hubo más del $15 \%$ de pérdida de participantes en uno de los grupos de intervención.

Por otro lado, las intervenciones realizadas no son de fácil comparación, ya que pocos estudios comparan un solo enfoque de tratamiento y en su mayoría utilizan un enfoque ecléctico. Por ejemplo, Brock et $\mathrm{al}^{45}$ compararon en pacientes en etapa aguda, los efectos a corto plazo del enfoque Bobath adicionando la práctica de actividades cotidianas y el tratamiento con énfasis en tareas funcionales; mientras que Tung, et al. ${ }^{44}$ compararon en pacientes crónicos los efectos de un enfoque ecléctico y el entrenamiento del paso de sedente a de pie.

Otras limitaciones encontradas evidencian diversidad de variables de salida o mediciones, Langhammer, et al. ${ }^{35} \mathrm{y}$ Vliet, et al. ${ }^{41}$ evaluaron los efectos del enfoque 
Bobath y del reaprendizaje motor en la rehabilitación temprana, sin embargo, las variables de salida evaluadas fueron diferentes (calidad de vida con el perfil de
Nottingham y tiempo de hospitalización, comparado con la distancia recorrida en la marcha 6 minutos y la EAM, respectivamente).

Tabla 1. Descripción de los ensayos clínicos controlados incluidos en la revisión.

\begin{tabular}{|c|c|c|c|c|}
\hline Autor-año & objetivo & población de estudio & variables & resultados \\
\hline $\begin{array}{l}\text { Langhammer, } \\
\text { et al. } 2000\end{array}$ & $\begin{array}{l}\text { Comparar los efectos de } \\
\text { dos enfoques de fisioterapia } \\
\text { sobre la rehabilitación } \\
\text { temprana post-ECV. }\end{array}$ & $\begin{array}{l}\mathrm{n}=61 \text { hospitalizados } \\
\text { ECV. } \\
\text { Mujeres: } 25 \\
\text { Hombres: } 36 \\
\text { Edad: } 78 \pm 9 \text {; rango (49- } \\
\text { 95). }\end{array}$ & $\begin{array}{l}\text { Salida } \\
\text { Función motora (Escala de } \\
\text { Evaluación Motora), funcionalidad } \\
\text { (Índice de Barthel (IB)), calidad de } \\
\text { vida (Perfil de Salud de Nottingham), } \\
\text { tiempo de hospitalización. } \\
\text { Explicatorias } \\
\text { G1: Programa de Reaprendizaje } \\
\text { Motor (PRM). } \\
\text { G2: Enfoque Bobath, }\end{array}$ & $\begin{array}{l}\text { No hubo diferencias significativas } \\
\text { entre grupos }(p>0,05) \text {. } \\
\text { Diferencias significativas en ambos } \\
\text { grupos }(p=0,0001) \text {. } \\
\text { Tiempo de Hospitalización ( } \overline{\mathrm{X}} \text { PRM } \\
21 \text { días vs Bobath } 34 \text { días, } p=0,008) \text {. }\end{array}$ \\
\hline $\begin{array}{l}\text { Green, et al. } \\
2002\end{array}$ & $\begin{array}{l}\text { Evaluar la efectividad de } \\
\text { una rutina de fisioterapia } \\
\text { comunitaria en pacientes } \\
\text { con dificultades en la } \\
\text { movilidad un año después } \\
\text { del inicio del ECV. }\end{array}$ & $\begin{array}{l}\mathrm{n}=170 \\
\text { Mujeres: } 75 \\
\text { Hombres: } 95 \\
\text { Edad: } \quad 71,5 \pm 8,7 \\
73,5 \pm 8,3 .\end{array}$ & $\begin{array}{l}\frac{\text { Salida }}{\text { Movilidad (Índice de Movilidad de }} \\
\text { Rivermead), velocidad de la marcha } \\
\text { (marcha } 10 \mathrm{~m} \text { ), funcionalidad } \\
\text { (Índice de Barthel (IB)), actividades } \\
\text { instrumentales (Índice de } \\
\text { Actividades de Frenchay). } \\
\text { Explicatorias } \\
\text { G1: Fisioterapia comunitaria. } \\
\text { G2: Control (no tratamiento). }\end{array}$ & $\begin{array}{l}3 \text { meses } \\
\text { Movilidad: } \mathrm{G} 1=11(8-12), \mathrm{G} 2=10(8- \\
12) \text {. } \\
\text { Velocidad de marcha: } 25,5 \pm 12,6 \mathrm{~m} / \\
\text { min y } 24,9 \pm 13,8 \mathrm{~m} / \mathrm{min} . \\
\text { Funcionalidad: } 18(16-19) \text { y } 18(16- \\
\text { 19). } \\
\text { Actividades: } 9(4-15) \text { y } 12(5-17) . \\
6 \text { meses } \\
\text { Movilidad: G1=11(8-12), G2=10(8- } \\
\text { 12). } \\
\text { Velocidad de marcha: } 26 \pm 13,1 \text { y } \\
25,3 \pm 13,6 . \\
\text { Funcionalidad: } 18(16-19) \text { y } 18(16- \\
19) . \\
\text { Actividades: } 11(5-18) \text { y } 15(6-21) .\end{array}$ \\
\hline $\begin{array}{l}\text { Kwakkel, et } \\
\text { al. } 2002\end{array}$ & $\begin{array}{l}\text { Investigar los efectos de } \\
\text { diferentes duraciones } \\
\text { de rehabilitación del } \\
\text { Miembro Superior (MS) e } \\
\text { Inferior (MI) en pacientes } \\
\text { hemipléjicos post-ECV. }\end{array}$ & $\begin{array}{l}\mathrm{n}=53 \text {, etapa aguda. } \\
\text { Mujeres: } 17 \\
\text { Hombres: } 36 \\
\text { Edad: } 62,1 \pm 10,6 \text {, } \\
64,3 \pm 10,6 \text { y } 60,8 \pm 10,6 .\end{array}$ & $\begin{array}{l}\text { Salida } \\
\text { Velocidad de la marcha (marcha } 10 \\
\text { m), funcionalidad (IB), índice de } \\
\text { motricidad, evaluación motora (Fugl } \\
\text { Meyer). } \\
\text { Explicatorias } \\
\text { G1: Férula de presión inflable en MS } \\
\text { y MI. } \\
\text { G2: Énfasis en MI } \\
\text { G3: Énfasis en MS }\end{array}$ & $\begin{array}{l}\text { Diferencias significativas en la } \\
\text { velocidad de marcha confortable en } \\
\text { el } \mathrm{G} 2 \text { comparado con } \mathrm{G} 1 \mathrm{y} \mathrm{G} 3 .(0,18 \\
\mathrm{m} / \mathrm{s}, p=0,02 ; 0,21 \mathrm{~m} / \mathrm{s}, p=0,03) . \\
\text { No hubo diferencias en la } \\
\text { funcionalidad y evaluación motora. }\end{array}$ \\
\hline $\begin{array}{l}\text { Fang, et al. } \\
2003\end{array}$ & $\begin{array}{l}\text { Investigar si la fisioterapia } \\
\text { temprana adicional mejora } \\
\text { la recuperación funcional } \\
\text { en pacientes com ECV. }\end{array}$ & $\begin{array}{l}n=156, \text { etapa aguda. } \\
\text { G1 }(n=50) \\
\text { G2 }(n=78) \\
\text { Mujeres: } 51 \\
\text { Hombres: } 77 \\
\text { Edad: } \quad 65,5 \pm 10,9 \\
61,8 \pm 10,9 .\end{array}$ & 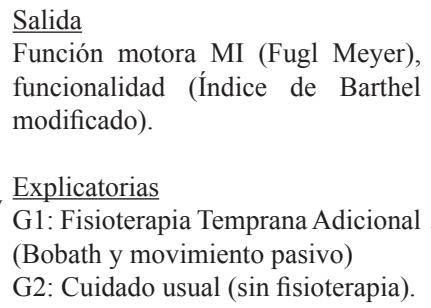 & $\begin{array}{l}\text { Pérdidas de más del } 20 \% \text { de la } \\
\text { muestra. } \\
\text { Diferencias significativas en todos } \\
\text { los grupos }(p<0,05) \text {. } \\
\text { No hubo diferencias entre los grupos } \\
\text { en la función motora y funcionalidad. }\end{array}$ \\
\hline $\begin{array}{l}\text { Duncan, et al. } \\
2003\end{array}$ & $\begin{array}{l}\text { Determinar si un programa } \\
\text { de ejercicio progresivo } \\
\text { y estructurado para } \\
\text { pacientes con ECV agudo } \\
\text { produce mayores efectos } \\
\text { que el cuidado usual y la } \\
\text { recuperación espontánea. }\end{array}$ & $\begin{array}{l}n=100 \\
\text { G1 }(n=44) \\
\text { G2 }(n=48) \\
\text { Mujeres: } 42 \\
\text { Hombres: } 50 \\
\text { Edad: } \quad 68,5 \pm 9 \\
70,2 \pm 11,4 .\end{array}$ & $\begin{array}{l}\text { Salida } \\
\text { Recuperación motora (Fugl Meyer), } \\
\text { fuerza isométrica de la dorsiflexión y } \\
\text { extensión de rodilla (DI), volumen de } \\
\text { oxigeno pico, velocidad de la marcha } \\
\text { (marcha } 10 \mathrm{~m} \text { ), distancia recorrida } \\
\text { (marcha } 6 \text { minutos), balance (EB } \\
\text { Berg), alcance funcional. } \\
\text { Explicatorias } \\
\text { G1: Fisioterapia } \\
\text { G2: Cuidado usual }\end{array}$ & 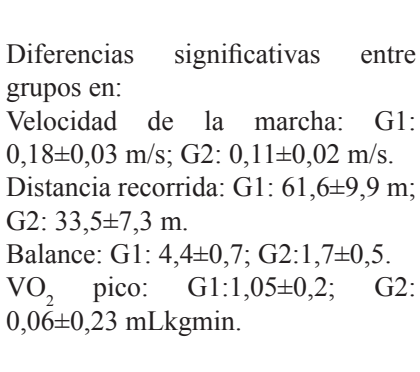 \\
\hline
\end{tabular}




\begin{tabular}{|c|c|c|}
\hline Autor-año & población de estudio & resultados \\
\hline $\begin{array}{l}\text { Eich, et al. } \\
2004\end{array}$ & $\begin{array}{l}\text { Evaluar el efecto } \\
\text { inmediato y a largo plazo } \\
\text { de la banda sinfín más el } \mathrm{n}=50 \\
\text { entrenamiento de marcha } \mathrm{G} 1: 25 \text { y } \mathrm{G} 2: 25 \text {. } \\
\text { de Bobath en pacientes con Mujeres: } 17 \\
\text { ECV agudo comparado con Hombres: } 33 \\
\text { entrenamiento de marcha Edad: } 62,4 \pm 4,8 \text { y } 64 \pm 6 \text {. } \\
\text { de Bobath solamente. }\end{array}$ & 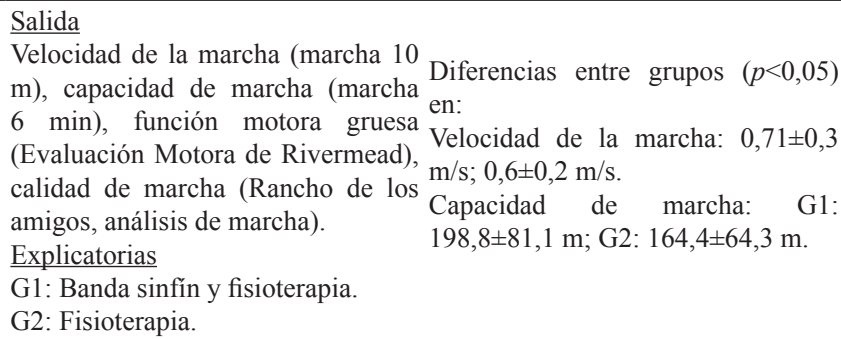 \\
\hline $\begin{array}{l}\text { Vliet, et al. } \\
2005\end{array}$ & $\begin{array}{l}\text { El objetivo del presente } \mathrm{n}=120, \quad \text { etapa } \\
\text { estudio fue evaluar si el aguda }(0-2 \text { semanas } \\
\text { tratamiento con el enfoque evolución). } \\
\text { Bobath o el Reaprendizaje } \mathrm{G} 1: 60 \text { y G2:60 } \\
\text { Motor es más efectivo en la Mujeres: } 60 \\
\text { mejora de las habilidades } \text { Hombres: } 60 \\
\text { de movimiento y la Edad: } 73,3 \pm 10,4 \text { y } \\
\text { independencia funcional } 75 \pm 9,1 .\end{array}$ & $\begin{array}{l}\frac{\text { Salida }}{\text { Desempeño motor (E. motora de }} \\
\text { Rivermead), función motora (Escala } \\
\text { de Evaluación Motora), distancia } \\
\text { recorrida (marcha } 6 \text { min), tono No hubo diferencias significativas } \\
\text { muscular (EAM), funcionalidad entre grupos }(p>0,05) \text {. } \\
\text { (IB). } \\
\text { Explicatorias } \\
\text { G1: Enfoque Bobath. } \\
\text { G2: Reaprendizaje Motor. }\end{array}$ \\
\hline
\end{tabular}

Diferencias significativas en cada grupo.
Wang, et al. 2005

Pohl, et al. 2007

Tung, et al. 2010

Brock, et al. 2011
Investigar los efectos del entrenamiento de sedente a de pie sobre el desempeño con ECV. $\underline{\text { Salida }}$

$\mathrm{n}=44$ etapa aguda Alteración motora (Evaluación de (G1:23 y G2:21).

Investigar la efectividad Mujeres: 16
del enfoque Bobath y el del enfoque Bobath y el Hombres: 28 tratamiento ortopédico en diferentes etapas motora en pacientes con $\mathrm{ECV}$. Edad: $\quad 59,3 \pm 12,2$, salud (Escala de Impacto en ECV) $63,8 \pm 13,1 ; 53,9 \pm 11,1$, $62,4 \pm 11,6$.

\section{Explicatorias}

G1: Tratamiento ortopédico.

G2: Enfoque Bobath.
Diferencias entre grupos en:

Grupo espasticidad

Función motora: $15,3 \pm 4,5 ; 18,8 \pm 5,8$

$(p=0,01)$.

Impacto ECV: $15,4 \pm 4,2 ; 18,9 \pm 5,1$ $(p=0,02)$.

Grupo recuperación relativa

Función motora: 25,5 $\pm 7 ; 24 \pm 4,8$

Balance: 46(23); 43(30).

Impacto ECV: 17 (9); 25 (12).

$\underline{\text { Salida }}$

Habilidad de marcha (Categoría de

Comparar el efecto $n=155$ en etapa aguda, Ambulación Funcional), velocidad y del entrenamiento $(\mathrm{G} 1: 77 \mathrm{y} \mathrm{G} 2: 78)$ distancia recorrida (marcha $10 \mathrm{~m}$ y 6 locomotor repetitivo Tiempo evolución: $0-2 \mathrm{~min}$ ), funcionalidad (IB), movilidad utilizando un caminador meses.

electromecánico en Mujeres: 51 combinación con Hombres: 104 fisioterapia y el efecto de la Edad: $12,3 \pm 12$ fisioterapia sola. $\quad 64 \pm 11,6$.
Diferencia entre grupos $(\mathrm{p}<0,05)$.

Velocidad de marcha: $0,44 \pm 0,47 \mathrm{~m} / \mathrm{s}$; $0,32 \pm 0,36 \mathrm{~m} / \mathrm{s}$

(IM Rivermead), potencia motora del MI comprometido (Índice de Distancia recorrida: 134,4 $\pm 125,5 \mathrm{~m}$; Motricidad).

y Explicatorias

Entrenamiento locomotor y

Movilidad: 9 (5-12); $11(7,5-13)$.

fisioterapia.

G2: Fisioterapia.

$\underline{\text { Salida }}$

$\mathrm{n}=32(\mathrm{G} 1: 16$ y G2:16). Balance de pie (Sistema Master Diferencias entre grupos $(p<0,05)$ Tiempo evolución: Balance), balance en tareas en:

$12,8 \pm 12,3$ y $26,9 \pm 16$ funcionales $(\mathrm{EBB})$, fuerza extensora Balance de pie (Control direccional: meses.

de cadera, rodilla y plantiflexores del balance en individuos

Mujeres: 12

Hombres: 20

(Dinamómetro manual),

Explicatorias

Edad: $\quad 57,7 \pm 14,1 \quad$ y G1: Fisioterapia (control)

$51 \pm 12,1$.

$62,6 \pm 26,1 \% ; 62,8 \pm 29,7 \%$ ).

Duración del PSP: $2,1 \pm 0,7 \mathrm{~s} ; 2,5 \pm 1,4 \mathrm{~s}$.

Fuerza extensora de

Cadera MI afectado: 22,6 $\pm 8,4 \%$; G2: Fisioterapia y entrenamiento de $22,8 \pm 7,2 \%$. sedente a de pie.

Comparar los efectos a ${ }_{n=26}(\mathrm{G} 1: 12$ y G2:14). Salida Diferencias significativas en cada corto plazo del enfoque

Bobath adicionando $\begin{array}{lll}60,3 \pm 24 & \text { y } & 63,6 \pm 25,9\end{array}$ adaptada), velocidad de la marcha

la práctica de tareas y $60,3 \pm 24$ y 63,6 25,9 (marcha 10 metros), balance (EBB),

la práctica de tarea días.

estructurada sobre la Mujeres: 7

capacidad de caminar en Hombres: 19

diferentes entornos post- Edad: 61,3 \pm 13

ECV.
Explicatorias funcionales.

y G2: Fisioterapia basada en tareas Velocidad de marcha: $56,8 \pm 28,3 \mathrm{~m} /$ funcionales. $\mathrm{min} ; 36,2 \pm 27,9 \mathrm{~m} / \mathrm{min}$.
G1: Enfoque Bobath y tareas Distancia recorrida:192,5 $\pm 113,5 \mathrm{~m}$; 
En seis artículos revisados se encontró que los tratamientos realizados incluían un enfoque fisioterapéutico ecléctico. Dentro de este tratamiento convencional, se manejan estímulos sensoriales táctiles, auditivos, visuales y propioceptivos (estiramientos, resistencia externa entre otros), que hacen parte del enfoque $\operatorname{Rood}^{23}$. Además, el estiramiento muscular es una técnica de tratamiento utilizada en los enfoques anteriormente mencionados, con un nivel de evidencia B (recomendación favorable) $)^{2}$, el cual ha sido ampliamente utilizado para prevenir contracturas y mejorar el arco de movilidad articular ${ }^{27}$.

Los ejercicios de estiramiento pueden ser ejecutados en un gran número de modalidades, los cuales incluyen el estiramiento manual pasivo (es desarrollado por otra persona y el paciente no participa activamente); el estiramiento activo (el paciente inicia y/o mantiene el estiramiento) y el posicionamiento que es utilizado mediante posturas inhibitorias durante el tratamiento ${ }^{48,49}$.

El enfoque más utilizado en fisioterapia para el tratamiento de pacientes post-ECV es el Neurodesarrollante o Bobath, el cual tiene un nivel de evidencia A (extremadamente recomendable) $)^{2,34}$. Durante la revisión se encontraron cinco estudios $^{35,38,41,42,45}$ que incluyeron dicho enfoque, sin embargo en dos de ellos se comparó su efecto con un programa de reaprendizaje motor ${ }^{35,41}$, uno con el tratamiento ortopédico ${ }^{42}$, uno con un enfoque ecléctico con énfasis en tareas funcionale ${ }^{45}$ y otro con un grupo sin tratamiento ${ }^{38}$. En una revisión de Kollen, et al. ${ }^{50}$ se evaluó la evidencia en 16 ensayos clínicos controlados sobre la eficacia del enfoque Bobath en pacientes post$\mathrm{ECV}$, comparado con otros enfoques neuromusculares y el tratamiento convencional. Aunque se evidenció mejoría con las intervenciones realizadas, no hubo cambios significativos entre los grupos en el control sensoriomotor de los MMSS y MMII, en las tareas funcionales y la calidad de vida $(p>0,05)$.

El enfoque Bobath enfatiza en la facilitación sensorial en el lado más afectado utilizando puntos clave de control, posturas dinámicas para el trabajo del mecanismo reflejo postural y Patrones Inhibitorios de Movimiento (PIM) para el control del tronco y la extremidad afectada ${ }^{51,52}$.

Solamente en el estudio de Duncan, et al. ${ }^{39}$ se encontró de forma específica el manejo con las técnicas de FNP. En este estudio se comparó un programa progresivo de fisioterapia con el cuidado usual en pacientes post-ECV. Aunque dentro del tratamiento convencional se utilizan estas técnicas, en los estudios revisados no se muestra el protocolo de intervención de forma detallada.
De forma general, las técnicas de FNP son métodos utilizados para promover o acelerar respuestas específicas del sistema neuromuscular por medio de combinaciones de patrones de movimiento en masa y en diagonales que se relacionan con movimientos llevados a cabo en diferentes actividades funcionales ${ }^{23}$. Siendo así, un enfoque multisensorial, que por medio de contactos manuales, comandos verbales y estímulos visuales busca fortalecer (facilitar) y relajar (inhibir) un grupo muscular en particular, músculos sinergistas o $\operatorname{antagonistas}^{52,53}$.

Los estudios revisados presentan sesgos de clasificación (falta de evaluación de las propiedades psicométricas a los instrumentos utilizados y de enmascaramiento); sesgos de selección (falta de homogeneidad de los grupos al inicio del estudio ${ }^{38,41}$ y de control del espectro de la enfermedad, ya que la etapa de cronicidad de los pacientes es variable) ${ }^{36}$ y de confusión (ningún estudio estableció el análisis de los resultados teniendo en cuenta variables de control y la falta de estandarización en los parámetros de intervención). Además, en algunos estudios hubo factores de confusión como en el trabajo de Green, et al. ${ }^{36}$ donde hubo un tiempo de intervención entre 3 y 13 semanas y no se especifican los parámetros de aplicación del tratamiento.

Tabla 2. Análisis de la calidad metodológica de los estudios.

Tabla 2. Análisis de la calidad metodológica de los estudios.

$\begin{array}{lllllllllllll}\text { Artículo } & 1 & 2 & 3 & 4 & 5 & 6 & 7 & 8 & 9 & 10 & 11 & \text { total }\end{array}$

\begin{tabular}{ccccccccccccc}
$\begin{array}{c}\text { Langhammer, } \\
\text { 2000 }\end{array}$ & 1 & 1 & 1 & 1 & 1 & 0 & 1 & 1 & 1 & 1 & 0 & 8 \\
Green, 2002 & 1 & 1 & 1 & 1 & 0 & 1 & 1 & 1 & 1 & 1 & 1 & 9 \\
Kwakkel, 2002 & 1 & 1 & 1 & 1 & 0 & 0 & 1 & 1 & 1 & 1 & 1 & 8 \\
Fang, 2003 & 1 & 1 & 1 & 0 & 0 & 1 & 1 & 0 & 1 & 1 & 0 & 6 \\
Duncan, 2003 & 1 & 1 & 1 & 1 & 0 & 0 & 1 & 1 & 1 & 1 & 1 & 8 \\
Eich, 2004 & 1 & 1 & 1 & 1 & 0 & 0 & 1 & 1 & 1 & 1 & 0 & 7 \\
Vliet, 2005 & 1 & 1 & 1 & 0 & 0 & 1 & 1 & 0 & 1 & 1 & 1 & 7 \\
Wang, 2005 & 1 & 1 & 1 & 1 & 0 & 0 & 1 & 1 & 1 & 1 & 0 & 7 \\
Pohl, 2007 & 1 & 1 & 1 & 1 & 0 & 0 & 0 & 1 & 1 & 1 & 0 & 6 \\
Tung, 2010 & 1 & 1 & 1 & 1 & 0 & 0 & 0 & 1 & 1 & 1 & 1 & 7 \\
Brock, 2011 & 1 & 1 & 1 & 1 & 0 & 0 & 1 & 1 & 1 & 1 & 1 & 8 \\
\hline
\end{tabular}

Otra de las características de los estudios que dificulta la comparación de los mismos es el número de intervenciones y los resultados contradictorios. Tung, et 
al. ${ }^{44}$ compararon el efecto del tratamiento convencional adicionándole un entrenamiento del paso de sedente a de pie (grupo experimental, 45 minutos) y el tratamiento convencional (grupo control, 30 minutos) tres veces por semana, durante cuatro semanas, evidenciando una mejoría significativa en el balance estático, dinámico y la fuerza de los músculos extensores de cadera en el MI más comprometido; Langhammer, et al..$^{35}$ compararon los efectos del tratamiento con el enfoque Bobath y el enfoque orientado a las tareas durante tres meses de intervención (5 días/semana, 40 minutos sesión) y no encontraron diferencias estadísticamente significativas entre los dos grupos $(p>0,05)$.

A continuación, se presenta el análisis de los parámetros de intervención revisados en la literatura.

\section{Parámetros de las intervenciones terapéuticas}

Hay un gran debate acerca de la cantidad de fisioterapia necesaria en personas post-ECV o si existe un umbral mínimo para conseguir una mejoría específica, sin embargo, esta pregunta se encuentra aún sin responder. En la literatura hay poca evidencia para guiar con precisión los parámetros de intervención, las guías de fisioterapia del Reino Unido consideran que un paciente con ECV debería recibir un mínimo de 45 minutos de terapia por sesión, 5 días por semana, teniendo en cuenta la tolerancia del paciente y priorizando los objetivos de rehabilitación ${ }^{2,54}$.

Los parámetros de intervención fisioterapéutica de los estudios revisados se encuentran en la tabla 3.

Tabla 3. Parámetros de las intervenciones terapéuticas (frecuencia, duración e intensidad).

\begin{tabular}{|c|c|c|c|}
\hline \multicolumn{4}{|c|}{ Parámetros de aplicación del tratamiento } \\
\hline Autor & Grupo & Frecuencia & Duración e intensidad \\
\hline Langhamme, et al. 2000 & $\begin{array}{l}\text { G1: Programa de Reaprendizaje } \\
\text { Motor. } \\
\text { G2: Enfoque Bobath. }\end{array}$ & $\begin{array}{l}5 \text { veces/semana, durante el tiempo de } \\
\text { hospitalización. }\end{array}$ & 40 minutos \\
\hline Green, et al. 2002 & $\begin{array}{l}\text { G1: Fisioterapia comunitaria. } \\
\text { G2: No tratamiento. }\end{array}$ & Entre 3 y 13 semanas por paciente. & No se especifican parámetros. \\
\hline Kwakkel, et al. 2002 & $\begin{array}{l}\text { G1: Férula de presión inflable en } \\
\text { MS y MI. } \\
\text { G2: Énfasis en MI } \\
\text { G3: Énfasis en MS }\end{array}$ & 5 veces/semana, durante 20 semanas. & $\begin{array}{l}30 \text { minutos con cada } \\
\text { intervención }+30 \text { minutos } \\
\text { adicionales de ejercicios de } \\
\text { MMSS y MMII. }\end{array}$ \\
\hline Fang, et al. 2003 & $\begin{array}{l}\text { G1: Fisioterapia (Bobath y } \\
\text { movimiento pasivo) } \\
\text { G2: No tratamiento. }\end{array}$ & 5 veces/semana, durante 4 semanas. & 45 minutos \\
\hline \multirow[t]{2}{*}{ Duncan, et al. 2003} & G1: Fisioterapia & 3 veces/semana, durante 12 semanas. & 90 minutos \\
\hline & G2: Cuidado usual & Recomendaciones 1 vez cada 2 semanas. & \\
\hline \multirow{2}{*}{ Eich, et al. 2004} & G1: Fisioterapia y Banda sinfín. & $\begin{array}{l}7 \text { veces/semana, durante } 30 \text { días } \\
\text { consecutivos. }\end{array}$ & $\begin{array}{l}60 \text { minutos }(15 \% \text { del soporte } \\
\text { de peso corporal) }\end{array}$ \\
\hline & G2: Fisioterapia. & $\begin{array}{l}7 \text { veces/semana, durante } 30 \text { días } \\
\text { consecutivos. }\end{array}$ & 60 minutos \\
\hline Vliet, et al. 2005 & $\begin{array}{l}\text { G1: Enfoque Bobath. } \\
\text { G2: Reaprendizaje Motor. }\end{array}$ & No se especifica. & Mediana 23 (13-32) minutos. \\
\hline Wang, et al. 2005 & $\begin{array}{l}\text { G1: Tratamiento ortopédico. } \\
\text { G2: Enfoque Bobath. }\end{array}$ & 5 veces/semana, durante 4 semanas. & 40 minutos \\
\hline Pohl, et al. 2007 & $\begin{array}{l}\text { G1: Fisioterapia y Entrenamiento } \\
\text { locomotor. } \\
\text { G2: Fisioterapia. }\end{array}$ & 5 veces/semana, durante 4 semanas. & 45 minutos \\
\hline \multirow[b]{2}{*}{ Tung, et al. 2010} & G1: Fisioterapia. & 3 veces/semana, durante 4 semanas. & 30 minutos \\
\hline & $\begin{array}{l}\text { G2: Fisioterapia y entrenamiento de } \\
\text { sedente a de pie. }\end{array}$ & 3 veces/semana, durante 4 semanas. & 45 minutos \\
\hline Brock, et al. 2011 & $\begin{array}{l}\text { G1: Enfoque Bobath y tareas } \\
\text { funcionales. } \\
\text { G2: Fisioterapia basada en tareas } \\
\text { funcionales. }\end{array}$ & 3 veces/semana, durante 2 semanas. & 60 minutos \\
\hline
\end{tabular}


En esta revisión, se encontró gran variabilidad en el tiempo de duración de la sesión de tratamiento, desde 23 minutos hasta 90 minutos, siendo el tiempo de intervención que más se repitió de 45 minutos $^{38,43,44}$. Tung, et al. ${ }^{44}$ utilizaron tiempos diferentes de intervención en cada grupo, 30 minutos en el grupo control y 45 minutos en el grupo experimental, aspecto que podría ser favorable en el grupo tratado con mayor tiempo durante la sesión, sin embargo los dos grupos fueron similares en la evaluación del balance y fuerza muscular, aspecto que puede estar influenciado por el tipo de pruebas o herramientas utilizadas en las mediciones. Del mismo modo, la frecuencia del tratamiento fue más variable, en tres estudios se llevó a cabo cinco veces por semana durante cuatro semanas, mientras en el resto osciló entre tres a siete veces por semana, con un total de dos hasta 20 semanas de tratamiento.

Se ha evidenciado que una intervención temprana en la rehabilitación del paciente puede mejorar el proceso de recuperación y minimizar la discapacidad funcional ${ }^{55}$. El tratamiento iniciado dentro de los primeros 20 días se ha asociado con una probabilidad significativamente alta de excelentes respuestas terapéuticas (OR: 6,11: IC $95 \% 2,03$ a 18,36$)^{56}$. Del mismo modo, en la comparación de estudios respecto a la intensidad del tratamiento, se ha encontrado mejores resultados cuando la terapia se lleva a cabo durante los primeros seis meses post-evento $(p=0,001)^{33}$.

Kwakkel, et al. ${ }^{57}$ utilizaron un enfoque ecléctico con énfasis en la extremidad inferior y superior, obteniendo mejores resultados para la habilidad en las AVD, marcha y destreza, comparado con el grupo control (férula inflable) $(p<0,05)$, mientras que el grupo de entrenamiento con énfasis en miembro superior solo mostró mejoría en la destreza, con lo cual se puede interpretar que una mayor intensidad en la rehabilitación de la extremidad inferior mejora la recuperación funcional.

\section{Efectos fisiológicos del ejercicio terapéutico}

Los efectos fisiológicos del ejercicio terapéutico en personas con ECV pueden ser optimizados teniendo en cuenta aspectos como: el patrón de activación, el tiempo, la velocidad y tipo de contracción muscular ${ }^{26}$. Además, el fisioterapeuta debe modificar factores intrínsecos y extrínsecos que puedan incidir en su recuperación por medio de estrategias como: la aproximación y posicionamiento de los segmentos, las tomas de peso, comandos verbales claros, contactos adecuados, superficies de trabajo determinadas y la retroalimentación verbal y física que permitan al paciente evocar respuestas musculares específicas. El compromiso sensorial y cognitivo en algunos pacientes debe ser tenido en cuenta para la rehabilitación, por consiguiente, la importancia de focalizar la atención, brindar instrucciones claras y una retroalimentación de calidad $^{24,26,58}$.

El estiramiento muscular es una herramienta muy utilizada para el tratamiento de la espasticidad, el cual mejora las propiedades viscoelásticas de la unidad músculo-tendón, modula el tono muscular, mantiene o incrementa la extensibilidad de los tejidos blandos, reduce la posibilidad de contracturas y mejora la función motora ${ }^{59}$. Aunque hay pocos estudios que han evaluado las propiedades viscoelásticas después de intervenciones fisioterapéuticas, uno de los resultados que demuestran los efectos biomecánicos y neurales es el aumento en el Rango de Movilidad Articular y la disminución de la resistencia al movimiento pasivo ${ }^{60}$.

Gao, et al. ${ }^{61}$ investigaron los cambios en las propiedades biomecánicas activas y pasivas de la unidad músculotendón en sujetos saludables ( $\bar{x} 53,4 \pm 18,1$ años de edad) y con ECV en etapa crónica ( $\bar{x}$ 55,2 $\pm 9,9$ años de edad), durante la aplicación de estiramiento estático de la musculatura plantiflexora a una velocidad de $12 \%$, con una duración de 60 minutos (12 ciclos en secuencia de cinco minutos). Se evidenció una disminución significativa dentro del grupo de pacientes con ECV en el torque de resistencia a la dorsiflexión (pre: 11,9 $\pm 3,2 \mathrm{Nm}$ y post: $10,5 \pm 3,3 \mathrm{Nm}, p=0,009$ ); al igual que en la rigidez articular (pre: $0,60 \pm 0,13 \mathrm{Nm} /{ }^{\circ} \mathrm{y}$ post: $\left.0,49 \pm 0,14 \mathrm{Nm} /{ }^{\circ}, p<0,05\right)$. En este estudio el grupo control fue utilizado para comparar las variables antes de la intervención, ya que posterior al estiramiento se realizó el análisis solamente dentro del mismo grupo.

Bressel, et al. ${ }^{62}$ estudiaron 10 pacientes post-ECV ( $\overline{\mathrm{X}}$ $64,6 \pm 8,76$ años) el efecto inmediato durante 30 minutos de estiramiento estático y cíclico sobre los músculos plantiflexores utilizando el Dinamómetro Isocinético Kin-Com, encontrando diferencias significativas en la resistencia al movimiento pasivo y en el porcentaje de rigidez (razón torque-ángulo) en cada uno de los grupos $(p<0,05)$, sin embargo el grupo intervenido con estiramiento estático mostró un aumento del $53 \%$ en el torque de relajación $(p=0,01)$, lo cual demuestra la mejoría en la movilidad articular.

En la literatura incluida en esta revisión, no hay ECC de los enfoques terapéuticos mencionados que evalúen variables biomecánicas y neurofisiológicas (ej. Reflejo $\mathrm{H}$ ), ya que se ha focalizado en mediciones funcionales, 
de movilidad articular, fuerza y tono muscular, entre otras. Del mismo modo, hay pocos estudios que evalúan el efecto del estiramiento sobre la excitabilidad refleja en los MMSS o MMII, los cuales son realizados por medio de dispositivos mecánicos o ferulaje, herramientas que simulan los posibles efectos del estiramiento manual ${ }^{48}$.

Los mecanismos por los cuales estos enfoques terapéuticos podrían disminuir la espasticidad, están relacionados con el aumento en la flexibilidad del tejido conectivo circundante, la regulación central (corteza motora primaria por medio del tracto corticoespinal) y periférica del tono muscular ${ }^{62}$ Figura 2.

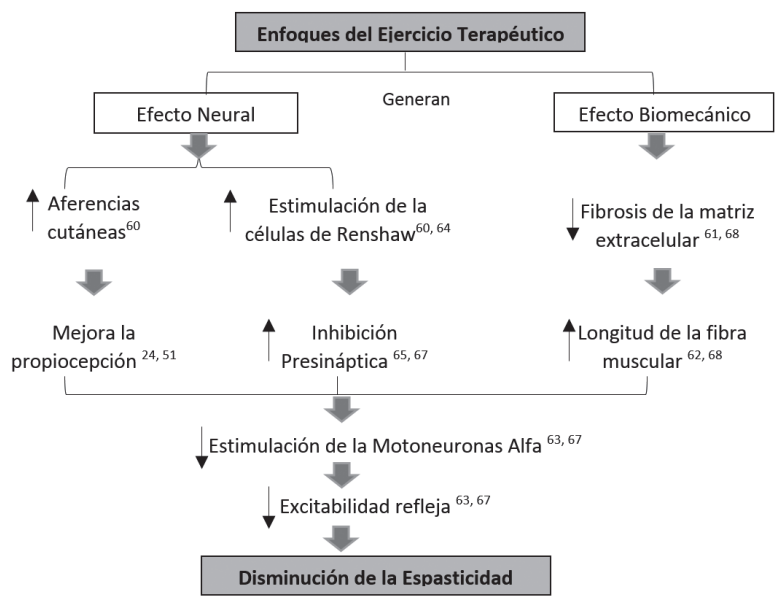

Figura 2. Mecanismos fisiológicos por los cuales los enfoques del ejercicio terapéutico disminuyen la espasticidad en personas con ECV.

Las respuestas periféricas se dan por la estimulación de mecanorreceptores cutáneos y musculares como los corpúsculos de pacini, terminaciones de Ruffini y el OTG, que emiten información por las fibras aferentes $I b$ las cuales hacen sinapsis con una interneurona inhibitoria de las motoneuronas alfa para relajar la musculatura agonista. Se ha encontrado que la estimulación de las fibras aferentes $I b$ del OTG y II del HNM pueden estar relacionados con la disminución de la excitabilidad refleja, sin embargo, los estudios son controversiales ${ }^{63}$.

Otro de los posibles mecanismos de acción inhibitoria es la inhibición presináptica, la cual puede deberse a la activación y despolarización de las fibras Ia generada por la activación de receptores GABA que permiten la apertura de canales de cloro en la terminal, lo cual disminuye la conductancia de la membrana, disminuyendo así, la liberación del neurotransmisor sobre la hendidura sináptica. El neurotransmisor GABA induce a que la despolarización se prolongue en la membrana presináptica; esta acción no previene el potencial de acción, sin embargo, disminuye la despolarización de la membrana postsináptica, reduciendo el estímulo excitatorio de las motoneuronas alfa $^{63-65}$.

Un hallazgo en la reducción de la excitabilidad refleja de las motoneuronas alfa se ha evidenciado con la disminución de la amplitud del Reflejo de Hoffmann (reflejo $\mathrm{H}$ ) después de la aplicación del estiramiento mantenido. El reflejo H es una medición neurofisiológica indirecta de la espasticidad que evalúa la excitabilidad de las fibras Ia del Huso Neuromuscular en el reflejo monosináptico, el cual es estimulado eléctricamente ${ }^{66}$. Los posibles mecanismos por los cuales se disminuye este reflejo se relacionan con la inhibición de las motoneuronas alfa, por medio de la activación de las fibras aferentes Ib del OTG o con la estimulación de las fibras aferentes tipo $\mathrm{II}^{62,64,67,68}$.

Otro hallazgo que se encuentra relacionado con la reducción de la excitabilidad refleja de las motoneuronas alfa después de una intervención es la disminución del tono muscular, ya que el incremento de la excitabilidad de dichas motoneuronas es un factor que contribuye a la espasticidad ${ }^{69}$, sin embargo, la evaluación del tono muscular es una medición menos sensible debido a la subjetividad y falta de estandarización en su aplicación $^{4,70}$.

Tsai, et al. ${ }^{67}$ estudiaron en 17 personas post-ECV en etapa crónica ( $\bar{X}$ 56,7 $\pm 11,6$ años de edad) el efecto de una sola sesión de 30 minutos de estiramiento muscular sobre los plantiflexores en posición de pie por medio de una tabla inclinada. Se evidenció un aumento de la movilidad articular para la dorsiflexión de cuello de pie (pre $15,1^{\circ} \pm 7,5$ y post $20,1^{\circ} \pm 6,4 ; p<0,05$ ) y una disminución de la excitabilidad refleja (Razón H/M: pre $42,8 \% \pm 22,3$ y post $29,2 \% \pm 17,3 ; p<0,05)$.

Los enfoques terapéuticos buscan modular diferentes niveles neurales, estabilizar los procesos integradores centrales y la activación de vías aferentes por medio de la apertura de vasos colaterales en la región lesionada, la conexión de nuevas sinapsis, el crecimiento dendrítico, la reorganización funcional del área lesionada y la participación de áreas homólogas ${ }^{26,71,72}$.

La recuperación de la función y la reorganización de mecanismos neurales se encuentran influenciados por la repetición y la experiencia, aspectos claves en el aprendizaje motor y la adaptación neural. La significancia de los estímulos realizados durante el tratamiento fisioterapéutico convencional permite 
mantener una interacción del individuo con el ambiente con la finalidad de replicar un patrón de movimiento en diferentes entornos y obtener o mantener un nuevo aprendizaje $\mathrm{e}^{24,26,71,72}$.

\section{DISCUSIÓN}

\section{Principales hallazgos y calidad de la evidencia}

Esta revisión incluyó 11 estudios, con una calidad metodológica entre intermedia a alta, sin embargo, en ocho de los estudios no hubo enmascaramiento del fisioterapeuta tratante $y$ en dos no hubo enmascaramiento de los evaluadores; dos estudios compararon algún enfoque de intervención con un grupo control sin tratamiento y en uno de ellos no se encontraron diferencias significativas en la función motora y la funcionalidad. Dentro de las limitaciones metodológicas encontradas en los estudios revisados se destaca principalmente la ausencia del cálculo del tamaño de muestra, lo cual pudo afectar el poder del estudio para detectar las diferencias realmente importantes y disminuir la generación de los errores sistemáticos tipo I y tipo II.

Las intervenciones realizadas en los artículos incluidos no fueron de fácil comparación, ya que pocos estudios compararon un solo enfoque de tratamiento y en su mayoría utilizaron un enfoque ecléctico, sin que se pudiese determinar los efectos de un enfoque específico. Además, otras limitaciones encontradas evidencian muestras heterogéneas, carencia de protocolos estandarizados y diversidad de variables de salida o mediciones, las cuales dificultan la comparación entre los estudios.

Por otro lado, la evidencia relacionada con la dosis de fisioterapia es limitada, esta discrepancia puede estar relacionada con la calidad metodológica de los ensayos, selección de los pacientes, intensidad del tratamiento, diferencias en tipo y tiempo de intervención de la ECV y diferencias en la medición de los resultados ${ }^{33}$.

La administración de las sesiones de fisioterapia podría variar en función de la habilidad y entrenamiento del fisioterapeuta, por tal motivo la importancia de estandarizar las intervenciones ${ }^{73}$; además, la evidencia de la eficacia de los tratamientos ha sido retrasada, tal vez debido a las dificultades técnicas experimentadas al momento de delimitar los criterios de inclusión y exclusión, ya que la población es heterogénea, es decir, existe una gran variabilidad en la función ${ }^{21,73}$.
Una de las estrategias más fuertes para reducir la diversidad en la rehabilitación de la ECV es la investigación de la eficacia de las intervenciones, teniendo en cuenta que el investigador debe ser capaz de determinar cuál de las intervenciones disponibles producen el mejor efecto para un grupo de pacientes; ya que la idea básica es que las herramientas metodológicas y estadísticas, si se aplican correctamente, disminuirán los posibles riesgos de sesgos ${ }^{74}$.

Por lo anterior, los estudios en la rehabilitación física no deben limitarse a enfoques individuales, por el contrario, se debe complementar con los enfoques que ya están claramente definidos y basados en la evidencia ${ }^{34}$.

\section{CONCLUSIÓN}

Los enfoques del ejercicio terapéutico podrían ser efectivos en la disminución de la espasticidad de los MMII post-ECV aplicándose entre tres y cinco días por semana, durante 40 minutos. Sin embargo, estos resultados están soportados en estudios con limitaciones metodológicas y dificultades en su comparación. Por tal motivo es necesario seguir evaluando la eficacia de dichos enfoques, con el fin de mejorar la evidencia y plantear intervenciones complementarias al tratamiento fisioterapéutico y evitar las complicaciones propias de la patología.

\section{REFERENCIAS}

1. Organización Mundial de la Salud. Global status report on noncommunicable diseases 2010. Publications of the World Health. Switzerland. Report No.: ISBN 9789241564229.

2. Hancock N, Kilbride C. National clinical guideline for stroke. R Coll Physicians, UK. 2012.

3. Orozco J. Enfermedad Cerebro Vascular. In: Ministerio de la Protección Social Guías para manejo de urgencias Tomo III. 3rd ed. Bogota; 2009. 169-177.

4. Burridge JH, Wood DE, Hermens HJ, Voerman GE, Johnson GR, van Wijck F, et al. Theoretical and methodological considerations in the measurement of spasticity. Disabil Rehabil. 2005; 27(1-2): 69-80. DOI: http://dx.doi. org/10.1080/09638280400014592.

5. Bhimani RH, Anderson LC, Henly SJ, Stoddard SA. Clinical measurement of limb spasticity in adults. J Neurosci Nurs. 2011; 43(2): 104-115. DOI: https:// doi.org/10.1097/jnn.0b013e31820b5f9f.

6. Ward AB. A literature review of the pathophysiology and onset of post-stroke spasticity. Eur J Neurol. 
2012; 19(1): 21-27. DOI: https://doi.org/10.1111/ j.1468-1331.2011.03448.x.

7. Wissel J, Schelosky LD, Scott J, Christe W, Faiss JH, Mueller J. Early development of spasticity following stroke: A prospective, observational trial. J Neurol. 2010; 257(7): 1067-1072. DOI: https:// doi.org/10.1007/s00415-010-5463-1.

8. Sommerfeld DK, Eek EUB, Svensson AK, Holmqvist LW, Von Arbin MH. Spasticity after Stroke: Its Occurrence and association with motor impairments and activity limitations. stroke. 2004; 35(1): 134-139. DOI: https://doi.org/10.1161/01. str.0000105386.05173.5e.

9. Urban PP, Wolf T, Uebele M, Marx JJ, Vogt T, Stoeter $\mathrm{P}$, et al. Occurence and clinical predictors of spasticity after ischemic stroke. Stroke. 2010; 41(9): 2016-2220. DOI: https://doi.org/10.1161/ strokeaha.110.581991.

10. Shilt J, Seibert P, Kadyan V. Optimal management for people with severe spasticity. Degener Neurol Neuromuscul Dis. 2012; 2: 133-140. DOI: https:// doi.org/10.2147/dnnd.s16630.

11. Faist M, Berger W, Dietz V. Changes in mechanical muscle fibre properties may contribute to spastic muscle hypertonia. Ann Readapt Med Phys. 1999; 42(8): 493-496. DOI: https://doi.org/10.1016/ s0168-6054(00)87703-x.

12. Bhimani R, Anderson L. Clinical understanding of spasticity: implications for practice. Rehabil Res Pract. 2014; 2014(278175): 1-10. DOI: https://doi. org/10.1155/2014/279175.

13. Van De Port I, Kwakkel G, Schepers V, Lindeman E. Predicting mobility outcome one year after stroke: a prospective cohort study. J Rehabil Med. 2006; 38(4): 218-223. DOI: https://doi. org/10.1080/16501970600582930.

14. Kwakkel G, Kollen BJ. Predicting activities after stroke: What is clinically relevant? Int J Stroke. 2013; 8(1): 25-32. DOI: https://doi.org/10.1111/ j.1747-4949.2012.00967.x.

15. Martin A, Abogunrin S, Kurth H, Dinet J. Epidemiological, humanistic, and economic burden of illness of lower limb spasticity in adults: a systematic review. Neuropsychiatr Dis Treat. 2014; 10: 111-122. DOI: https://doi.org/10.2147/ndt. s53913.

16. Aggarwal D, Walia S, Noohu M. Effect of plantarflexor spasticity and ankle joint range of motion on sit to stand movement in stroke patients. Physiother Occup Ther J. 2013; 6(1): 5-13.

17. Lee Y, Her JG, Choi Y, Kim H. Effect of Ankle-foot orthosis on lower limb muscle activities and static balance of stroke patients Authors' Names. J Phys
Ther Sci. 2014; 26(2): 179-182. DOI: https://doi. org/10.1589/jpts.26.179.

18. Ada L, Vattanasilp W, O’Dwyer NJ, Crosbie J. Does spasticity contribute to walking dysfunction after stroke?. J Neurol Neurosurg Psychiatry. 1998; 64: 628-635. DOI: https://doi.org/10.1136/ jnnp.64.5.628.

19. Schmid Arlene A, Klar Yaggi H, Burrus N, McClain V, Austin C, Ferguson J, et al. Circumstances and consequences of falls among people with chronic stroke. J Rehabil Res Dev. 2013; 50(9): 1277-1285. DOI: https://doi.org/10.1682/jrrd.2012.11.0215.

20. Peppen R Van, Kwakkel G, Wood-Dauphinee S. The impact of physical therapy on functional outcomes after stroke: what's the evidence? Clin Rehabilitation. 2004; 18: 833-862. DOI: doi/ abs/10.1191/0269215504cr843oa.

21. Pollock A, Baer G, Langhorne P, Pomeroy V. Physiotherapy treatment approaches for the recovery of postural control and lower limb function following stroke: a systematic review. 2007; 21: 395-410. DOI: https://doi.org/10.1177/0269215507073438.

22. Chung B. Effect of different combinations of physiotherapy treatment approaches on functional outcomes in stroke patients: a retrospective analysis. Hong Kong Physiother J. 2014; 32(1): 21-27. DOI: https://doi.org/10.1016/j.hkpj.2013.11.001.

23. Trombly C. Occupational therapy for physical dysfunction. 4ed ed. United States of America: Williams \& Wilkins; 1995. 446-460.

24. Umphred D. Remediating Motor Control and Performance Through Traditional Therapeutic Approaches. En: Neurological Rehabilitation. 5ed ed. United States of America: Churchill Livingstone; 2007. 433.

25. O'Sullivan S, Schmit T. Improving functional outcomes in physical rehabilitation. Philadelphia: Davis Company; 2010. 25-40.

26. Carr J, Shepherd R. Neurological Rehabilitation. Optimizing Motor Performance. 2ed. Ed. Churchill Livingstone; 2010. 77-83.

27. Katalinic OM, Harvey LA, Herbert RD, Katalinic OM, Harvey LA, Herbert RD. Effectiveness of stretch for the treatment and prevention of contractures in people with neurological conditions: a systematic review. 2011; 91(1): 11-24. DOI: https://doi.org/10.2522/ptj.20100265.

28. Stokes M. Fisioterapia en la rehabilitación neurológica. 2 ed. Madrid: Elsevier; 2006.

29. Platz T, Eickhof G, Vuadens P. Platz T. Clinical scales for the assessment of spasticity, associated phenomena, and function: a systematic review of the literature. Disabil Rehabil. 2005; 27: 7-18. DOI: 
https://doi.org/10.1080/09638280400014634.

30. Lee M, Wong M, Tang F, Cheng P, Lin P. Comparison of balance responses and motor patterns during sit-to-stand task with functional mobility in stroke patients. Am J Phys Med Rehabil. 1997; 76(5): 401-410. DOI: https://doi.org/10.1097/00002060199709000-00011.

31. Teixeira L, Olney S, Brouwer B. Mecanismos e medidas de espasticidade. Fisioter Pesqui. 1998; 5: 4-19.

32. Voerman GE, Gregoric M, Hermens HJ. Neurophysiological methods for the assessment of spasticity: the Hoffmann reflex, the tendon reflex, and the stretch reflex. Disabil Rehabil. 2005; 27(1-2): 33-68. DOI: https://doi. org/10.1080/09638280400014600.

33. Kwakkel G, Van Peppen R, Wagenaar RC, Dauphinee SW, Richards C, Ashburn A, et al. Effects of augmented exercise therapy time after stroke: a meta-analysis. Stroke. 2004; 35(11): 2529-2536. DOI: https://doi.org/10.1161/01. str.0000143153.76460.7d.

34. Pollock A, Baer G, Campbell P, Choo P, Forster A, Morris J, et al. Physical rehabilitation approaches for the recovery of function and mobility following stroke. Cochrane Database Syst Rev. 2014; 4: CD001920. DOI: https://doi.org/10.1002/14651858. cd001920.pub3.

35. Langhammer B, Stanghelle JK. Bobath or motor relearning programme? A comparison of two different approaches of physiotherapy in stroke rehabilitation: a randomized controlled study. Clin Rehabil. 2000; 14(4): 361-369. DOI: https://doi. org/10.1191/0269215500cr338oa.

36. Green J, Forster A, Bogle S, Young J. Physiotherapy for patients with mobility problems more than 1 year after stroke: A randomised controlled trial. Lancet. 2002; 359(9302): 199-203. DOI: https://doi. org/10.1016/s0140-6736(02)07443-3.

37. Kwakkel G, Wagenaar RC. Effect of duration of upper- and lower-extremity rehabilitation sessions and walking speed on recovery of interlimb coordination in hémiplégie gait. Phys Ther. 2002; 82(5): 432-448. DOI: https://doi.org/10.1093/ $\mathrm{ptj} / 82.5 .432$.

38. Fang Y, Chen X, Li H, Lin J, Huang R, Zeng J. A study on additional early physiotherapy after stroke and factors affecting functional recovery. Clin Rehabil. 2003; 17(6): 608-617. DOI: https://doi. org/10.1191/0269215503cr655oa.

39. Duncan P, Studenski S, Richards L, Gollub S, Lai SM, Reker D, et al. Randomized clinical trial of therapeutic exercise in subacute stroke. Stroke. 2003;
34(9): 2173-2180. DOI: https://doi.org/10.1161/01. str.0000083699.95351.f2.

40. Eich H-J, Mach H, Werner C, Hesse S. Aerobic treadmill plus Bobath walking training improves walking in subacute stroke: a randomized controlled trial. Clin Rehabil. 2004; 18(6): 640-651. DOI: https://doi.org/10.1191/0269215504cr779oa.

41. van Vliet PM, Lincoln NB, Foxall A. Comparison of Bobath based and movement science based treatment for stroke: a randomised controlled trial. J Neurol Neurosurg Psychiatry. 2005; 76(4): 503-508. DOI: https://doi.org/10.1136/jnnp.2004.040436.

42. Wang RY, Chen HI, Chen CY, Yang YR. Efficacy of Bobath versus orthopaedic approach on impairment and function at different motor recovery stages after stroke: a randomized controlled study. Clin Rehabil. 2005; 19(2): 155-164. DOI: https://doi. org/10.1191/0269215505cr850oa.

43. Pohl M, Werner C, Holzgraefe M, Kroczek G, Mehrholz J, Wingendorf I, et al. Repetitive locomotor training and physiotherapy improve walking and basic activities of daily living after stroke: a single-blind, randomized multicentre trial (DEutsche GAngtrainerStudie, DEGAS). Clin Rehabil. 2007; 21(1): 17-27. DOI: https://doi. org/10.1177/0269215506071281.

44. Tung FL, Yang YR, Lee CC, Wang RY. Balance outcomes after additional sit-to-stand training in subjects with stroke: a randomized controlled trial. Clin Rehabil. 2010; 24(6): 533-542. DOI: https:// doi.org/10.1177/0269215509360751.

45. Brock K, Haase G, Rothacher G, Cotton S. Does physiotherapy based on the Bobath concept, in conjunction with a task practice, achieve greater improvement in walking ability in people with stroke compared to physiotherapy focused on structured task practice alone?: a pilot randomized controlled. Clin Rehabil. 2011; 25(10): 903-912. DOI: https://doi.org/10.1177/0269215511406557.

46. Verhagen AP, Vet HCW De, Bie RA De, Alphons GH. The Delphi List: a Criteria List for quality assessment of randomized clinical trials for conducting systematic reviews developed by delphi consensus. J Clin Epidemiol. 1998; 51(12): 1235-1241. DOI: http://doi.org/10.1016/S08954356(98)00131-0.

47. Olivo SA, Macedo LG, Gadotti IC, Fuentes J, Stanton T, Magee DJ. Scales to assess the quality of randomized controlled trials: a systematic review. Phys Ther. 2008; 88(2): 156-175. DOI: https://doi. org/10.2522/ptj.20070147.

48. Bovend'Eerdt TJ, Newman M, Barker K, Dawes H, Minelli C, Wade DT. The Effects of Stretching 
in spasticity: a systematic review. Arch Phys Med Rehabil. 2008; 89(7): 1395-1406. DOI: https://doi. org/10.1016/j.apmr.2008.02.015.

49. Roberts J, Wilson K. Effect of stretching duration on active and passive range of motion in the lower extremity. BMJ Open Sport Exerc Med. 1999; 33(4): 259-263. DOI: https://doi.org/10.1136/ bjsm.33.4.259.

50. Kollen BJ, Lennon S, Lyons B, Wheatley-Smith L, Scheper M, Buurke JH, et al. The effectiveness of the Bobath concept in stroke rehabilitation what is the evidence?. Stroke. 2009; 40(4): 89-97. DOI: https://doi.org/10.1161/strokeaha.108.533828.

51. Bobath B. Concepto y Principios del Tratamiento. En: Hemiplejía del adulto: Evaluación y tratamiento. 3ed ed. Buenos Aires: Panamericana; 1999; 70-79.

52. Dickstein R, Hocherman S, Pillar T, Shaham R. Three Exercise Therapy Approaches. J Am Phys Ther Assoc. 1986; 66(8): 1233-1238. DOI: 10.1093/ $\mathrm{ptj} / 66.8 .1233$.

53. Voss D, Ionta M, Myers B. Facilitación neuromuscular propioceptiva. patrones y técnicas. 3ed ed. Madrid: Panamericana; 2004.

54. De Wit, Putman K, Dejaeger E, Baert I, Berman P, Bogaerts K. Use of time by stroke patients: a comparison of four European rehabilitation centers. Stroke. 2005; 36(9): 1977-1983. DOI: https://doi. org/10.1161/01.str.0000177871.59003.e3.

55. Duncan PW, Zorowitz R, Bates B, Choi JY, Glasberg JJ, Graham GD, et al. Management of adult stroke rehabilitation care: a clinical practice guideline. Stroke. 2005; 36(9). e100-143. DOI: https://doi. org/10.1161/01.str.0000177871.59003.e3.

56. Paolucci S, Antonucci G, Grasso MG, Morelli D, Troisi E, Cairo P, et al. Early versus delayed inpatient stroke rehabilitation: a matched comparison conducted in Italy. Arch Phys Med Rehabil. 2000; 81(6): 695-700. DOI: https://doi.org/10.1016/ s0003-9993(00)90095-9.

57. Kwakkel G, Kollen BJ, Wagenaar RC. Therapy impact on functional recovery in stroke rehabilitation: a critical review of the literature. Physiotherapy. 1999;85(7): 377-391. DOI: https:// doi.org/10.1016/s0031-9406(05)67198-2.

58. McDowd J, Filion D, Pohl P, Richards L, Stiers W. Attentional abilities and functional outcomes following stroke. J Gerontol B Psychol Sci Soc Sci. 2003; 58: 45-53. DOI: https://doi.org/10.1093/ geronb/58.1.p45.

59. Selles RW, Li X, Lin F, Chung SG, Roth EJ, Zhang LQ. Feedback-controlled and programmed stretching of the ankle plantarflexors and dorsiflexors in stroke: effects of a 4-week intervention program.
Arch Phys Med Rehabil. 2005; 86(12): 2330-2336. DOI: https://doi.org/10.1016/j.apmr.2005.07.305.

60. Smania N, Picelli A, Munari D, Geroin C, Ianes P, Waldner A, et al. Rehabilitation procedures in the management of spasticity. Eur J Phys Rehabil Med. 2012; 26: 423-438. PMID:20927008.

61. Gao F, Ren Y, Roth EJ, Harvey R, Zhang LQ. Effects of repeated ankle stretching on calf muscletendon and ankle biomechanical properties in stroke survivors. Clin Biomech. 2011; 26(5): 516-522. DOI: https://doi.org/10.1016/j.clinbiomech.2010.12.003.

62. Bressel E, Mcnair PJ. The Effect of prolonged static and cyclic stretching on ankle joint stiffness, torque relaxation, and gait in people with stroke. Phys Ther. 2002; 82: 880-887. DOI: https://doi.org/10.1093/ $\mathrm{ptj} / 82.9 .880$.

63. Mauritz KH. Neurorehabilitation in spasticity. Postępy Psychiatr i Neurol 2004; 2(18): 41-47.

64. Robinson KL, McComas AJ, Belanger AY. Control of soleus motoneuron excitability during muscle stretch in man. J Neurol Neurosurg Psychiatry. 1982; 45(8): 699-704. DOI: https://doi.org/10.1136/ jnnp.45.8.699.

65. Lamy JC, Wargon I, Mazevet D, Ghanim Z, Pradat-Diehl P, Katz R. Impaired efficacy of spinal presynaptic mechanisms in spastic stroke patients. Brain. 2009; 132(3): 734-748. DOI: https://doi. org/10.1093/brain/awn310.

66. Kohan AH, Abootalebi S, Khoshnevisan A, Rahgozar M. Comparison of modified ashworth scale and Hoffmann reflex in study of spasticity. Acta Med Iran. 2010; 48(3): 154-157. PMID: 21137650.

67. Tsai KH, Yeh CY, Chang HY, Chen JJ. Effects of a single session of prolonged muscle stretch on spastic muscle of stroke patients. Proc Natl Sci Counc Repub China B. 2001; 25(2): 76-81. PMID:11370763.

68. Yeh CY, Chen JJJ, Tsai KH. Quantifying the effectiveness of the sustained muscle stretching treatments in stroke patients with ankle hypertonia. J Electromyogr Kinesiol. 2007;17(4): 453-61. DOI: https://doi.org/10.1016/j.jelekin.2006.07.001.

69. Robichaud JA, Agostinucci J, Vander Linden DW. Effect of air-splint application on soleus muscle motoneuron reflex excitability in nondisabled subjects and subjects with cerebrovascular accidents.Phys Ther. 1992; 72(3): 176-185. DOI: https://doi.org/10.1093/ptj/72.3.176.

70. Annaswamy T, Mallempati S, Allison SC, Abraham LD. Measurement of plantarflexor spasticity in traumatic brain injury. Am J Phys Med Rehabil. 2007; 86(5): 404-411. DOI: https://doi.org/10.1097/ 
phm.0b013e31804a7d 85 .

71. Doussoulin-Sanhueza MA. Como se fundamenta la neurrorehabilitación desde el punto de vista de la neuroplasticidad. Arch Neurociencias. 2011; 16(4): 216-222.

72. Plow EB, Carey JR, Nudo RJ, Pascual-Leone A. Invasive cortical stimulation to promote recovery of function after stroke. Am Hear Assoc. 2008; 144(5): 724-732. DOI: https://doi.org/10.1161/ strokeaha.108.540823.

73. Selzer ME, Zorowitz RD. Designing prospective, randomized, multicenter clinical trials of physical rehabilitation treatment modalities. Neurorehabil Neural Repair. 2003; 17: 135-136. DOI: https://doi. org/10.1177/0888439003257318.

74. Lettinga A, Reynders K, Mulder T, Mol A. Pitfalls in effectiveness research: a comparative analysis of treatment goals and outcome measures in stroke rehabilitation. Clin Rehabil. 2002; 16: 174-181. DOI: https://doi.org/10.1191/0269215502cr472oa. 